\title{
B7H3 regulates differentiation and serves as a potential biomarker and theranostic target for human glioblastoma
}

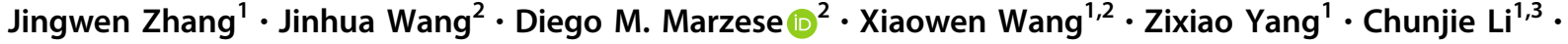 \\ Haibo Zhang ${ }^{1} \cdot$ Jinsen Zhang ${ }^{1}$. Clark C. Chen ${ }^{4}$. Daniel F. Kelly ${ }^{5} \cdot$ Wei Hua ${ }^{1,2,3} \cdot$ Dave S. B. Hoon $^{2,6} \cdot$ Ying Mao $^{1,3,7,8}$
}

Received: 17 September 2018 / Revised: 9 February 2019 / Accepted: 18 February 2019 / Published online: 26 March 2019

(c) United States \& Canadian Academy of Pathology 2019

\begin{abstract}
B7H3 (CD276), a co-stimulator molecule of the cell surface B7 protein superfamily, is expressed on glioblastomas (GBM). Recently, B7H3 functions beyond immune costimulation have been demonstrated. However, the mechanisms underlying B7H3 function are diverse and not well understood. GBM tumors contain undifferentiated self-renewing cells, which confound therapeutic attempts. We investigated the role of B7H3 in the regulation of GBM cell differentiation and the regulatory pathways involved. Analysis of public databases (TCGA, Rembrandt, and GEO NCBI) and RNA sequencing were performed to explore the role of B7H3 in GBM. Knockdown and overexpression of B7H3, were used to verify the downstream pathway in vitro. Further studies in vivo were performed to support the new finding. Bioinformatics analysis identified a correlation between the expression of $\mathrm{B} 7 \mathrm{H} 3$, the expression of glioma self-renewing cell (GSC)-related genes, and MYC expression. These observations were verified by RNA-sequencing analyses in primary GBM cell lines. In vitro knockdown of B7H3-induced differentiation, associated with downregulation of SMAD6 (a TGF- $\beta$ pathway inhibitor) and enhancement of SMAD1 phosphorylation-induced SMAD4 expression. Importantly, activation of the TGF- $\beta$ pathway resulted in downregulation of MYC expression. In vivo assays conducted in a human GBM cell line xenograft mouse model demonstrated that B7H3 knockdown decreased MYC expression and inhibited tumor growth. B7H3 knockdown could regulate GBM differentiation by modulating MYC expression. So, B7H3 could serve as a potential theranostic target for the treatment of patients with GBM.
\end{abstract}

Prof. Ying Mao and Prof. Dave S.B. Hoon both contributed as senior authors

Supplementary information The online version of this article (https:// doi.org/10.1038/s41374-019-0238-5) contains supplementary material, which is available to authorized users.

$\triangle$ Wei Hua

hs_glioma@126.com

1 Department of Neurosurgery, Huashan Hospital, Fudan University, Shanghai, China

2 Department of Translational Molecular Medicine, John Wayne Cancer Institute (JWCI) at Providence Saint John's Health Center (SJHC), Santa Monica, CA, USA

3 Institutes of Biomedical Sciences, Fudan University, Shanghai, China

4 Department of Neurosurgery, University of Minnesota, Minneapolis, MN, USA

\section{Introduction}

Glioblastoma (GBM), a grade IV glioma, is the most aggressive and commonly occurring primary tumor of the central nervous system. Despite recent research and therapeutic advancements, the prognosis of GBM patients remains dismal [1,2]. Genomic and epigenetic profiling of GBM in The Cancer Genome Atlas (TCGA) has allowed a

5 Brain Tumor Center and Pituitary Disorders Program, JWCI at Providence SJHC, Santa Monica, CA, USA

6 Sequencing Center, JWCI at Providence SJHC, Santa Monica, CA, USA

7 State Key Laboratory of Medical Neurobiology, School of Basic Medical Sciences and Institutes of Brain Science, Fudan University, Shanghai, China

8 The Collaborative Innovation Center for Brain Science, Fudan University, Shanghai, China 
better understanding of the molecular mechanisms of this primary brain cancer [3]. However, only minimal improvements have been made in treating patients with GBM, and most grade II and III gliomas eventually succumb to recurrence or progression after initial response to comprehensive treatment. Glioma tumor self-renewing cells (GSCs) are a known subpopulation in GBM, and are believed to play a role in resistance to therapy and tumor progression [4]. Much effort has been made to discover the essence of GSCs and to find optimal therapeutic targets [5]. Our previous report demonstrated that MYC could be epigenetically switched on and off in GBM as a key transcriptional factor deciding tumorigenicity [6].

Recently, the B7 superfamily of proteins has been afforded increasing attention due to the success of novel FDA-approved B7 molecule-targeted antibody (Ab)-therapies. B7 protein receptor-ligand axis regulators include B7H1, B7H2, PD1, and PDL1, against which monoclonal $\mathrm{Ab}$ therapeutics have been used successfully to treat many different types of solid tumors [7, 8]. The underlying mechanism of this treatment is through blocking the respective receptor-ligand axis. $\mathrm{B} 7 \mathrm{H} 3$ (CD276), a poorly characterized member of the B7 superfamily, is a type I transmembrane protein originally detected in immune cells, such as dendritic cells and specific T-cell subsets [9, 10], as well as in a variety of human solid tumor cells, including melanoma [11], breast cancer [12], ovarian cancer [13], pancreatic cancer [14], and gliomas [15, 16]. B7H3-related studies in cancer patients have suggested a relationship with antitumor immune responses [16]. B7H3 has been considered as a third group of immune checkpoints [17]. However, the role and regulatory mechanism of $\mathrm{B} 7 \mathrm{H} 3$ in tumor immunity is controversial [18]. B7H3 may have a functional role in some solid tumors, as we have previously shown in melanoma [11]. Interestingly, the ablation of B7H3 positive cells from tumors with antibody-drug conjugates (ADCs) could impede the tumor vasculature [19]. So far, the mechanisms underlying $\mathrm{B} 7 \mathrm{H} 3$ regulation remain diverse and unclear.

In this study, we have examined the expression of the $\mathrm{B} 7 \mathrm{H} 3$ gene and protein in gliomas, as well as the expression of other genes in relation to $\mathrm{B} 7 \mathrm{H} 3$ expression. By manipulating $\mathrm{B} 7 \mathrm{H} 3$ expression we investigated the physiological tumor-related functions of B7H3, including proliferation, migration, invasion in GBM in vitro. Knockdown of B7H3 perturbed intracellular signaling, in particular through the TGF- $\beta$ pathway and MYC activation, and altered expression of GSC-related genes. Furthermore, B7H3 knockdown as a therapy in a mouse xenograft model was examined. In sum, this study demonstrates a role for B7H3 in GBM tumorigenesis, provides a mechanism for its action, and suggests that $\mathrm{B} 7 \mathrm{H} 3$ may be a theronostic marker that offers a novel therapeutic potential through regulation of GSC differentiation.

\section{Materials and methods}

\section{Cells and culture}

A172, U138, U251, and U87 cells were purchased from ATCC and tested by short tandem repeat (STR) DNA profile for authenticity. Primary GBM (GBM1-9) cell lines were established from fresh surgical specimens of WHO grade IV human GBM using enzymatic and mechanical disaggregation, as previously described [20]. All the cell line experiments were completed within 12 passages.

\section{Immunofluorescence and immunohistochemical (IHC) staining}

Immunofluorescence staining of for B7H3 (1:200, R\&D Systems, Inc., Minneapolis, MN) was conducted on A172, U138, and GBM2 cells as described previously [20]. Formalin-fixed and paraffin-embedded (FFPE) tissue of gliomas of different grades were provided by the Department of Neurosurgery, Huashan Hospital, Shanghai (WHO Grade I, $n=13$; Grade II, $n=17$; Grade III, $n=19$; Grade IV, $n=50$ ) and the Brain Tumor Center in Providence Saint John's Health Center (WHO Grade IV, $n=8$ ). These data are reported according to the REMARK criteria [21]. Immunohistochemical (IHC) staining was conducted as described previously [11]. The sections were incubated with goat anti-human B7H3 Ab (1:200, R\&D Systems, Inc., Minneapolis, MN), anti-Ki67 Ab (1:500, Abcam, Cambridge, MA), anti-human Nestin Ab (1:100 Abcam), anti-c-MYC Ab (monoclonal, 1:1000, Abcam) as primary antibody. Immune reactive score (IRS) was calculated as described [16], and IRS $>6$ was considered as $\mathrm{B} 7 \mathrm{H} 3^{\text {hi }}$ expression.

\section{Western blot}

Protein concentrations were determined using the Pierce BCA assay (Thermo Scientific, Rockford, IL) and western blot was performed as previously described [11]. Membranes were probed individually with primary polyclonal goat anti-human B7H3 Ab (1:1000, R\&D Systems), polyclonal rabbit anti-nestin $\mathrm{Ab}$ (1:1000, Millipore Sigma, Carlsbad, CA), monoclonal mouse anti-GFAP Ab (1:1000, R\&D Systems), polyclonal mouse anti-CNPase Ab (1:200, Chemicon, Temecula, CA), polyclonal mouse anti-Tuj1 Ab (1:200). SMAD1, pSMAD1, SMAD2/3, SMAD4, SMAD6 antibodies (rabbit monoclonal, 1:1000, Cell Signaling Technology, Danvers, MA) and c-MYC antibody (monoclonal, 1:1000, Abcam were also applied in the study. The western blot analysis of clinical frozen specimens was performed in Shanghai using the enhanced chemiluminescence analysis system (Amersham 
Biosciences, Piscataway, NJ) according to the manufacturer's protocol.

\section{Flow cytometry analysis and fluorescence-activated cell sorting}

Single-cell suspensions of A172 and GBM2 cell lines were incubated with anti-B7H3, anti-A2B5 (rat monoclonal, Sigma Millipore), anti-Nestin, and anti-GFAP Ab diluted in PBS for $1 \mathrm{~h}$ at $4{ }^{\circ} \mathrm{C}$. After washing with PBS, cells were incubated with the secondary $\mathrm{Ab}$ for $30 \mathrm{~min}$. Flow cytometric analysis was performed using the FACSVerse System (BD Biosystems, Piscataway, NJ). Cells were sorted according to their expression of A2B5, a marker of GBM progenitor or GSC cells. For fluorescence-activated cell sorting (FACS), cells were washed with PBS containing $0.2 \%$ bovine serum albumin and incubated with Fc-block at $37^{\circ} \mathrm{C}$ for $30 \mathrm{~min}$. Anti-A2B5-PE Ab (Miltenyi Biotech, Auburn, CA) was then added to the cells and incubated at $37^{\circ} \mathrm{C}$ for $1 \mathrm{~h}$. Before sorting, the cells were washed three times. Sorting was carried out using a FACSCanto II flow cytometric machine (BD Biosystems, San Jose, CA). A2B5 positive and negative fractions were collected through different channels for further experiments.

\section{Overexpression and knockdown of B7H3 in GBM cells}

B7H3 expression plasmids (Origene, Rockville, MD) were delivered into U138 cells (which have low expression of B7H3) with JetPrime transfection agent (Polyplus, Berkeley, CA) for $6 \mathrm{~h}$, then successfully transfected cells were selected using $800 \mu \mathrm{g} / \mathrm{mL}$ geneticin (Gibco-BRL, Grand Island, NY) for 4 weeks to obtain stable clones.

B7H3 shRNAs (Sigma Aldrich, St Louis, MI) and B7H3 siRNAs (Thermo Scientific Dharmacon; cat\#J007813-11, J-007813-12) were transfected into A172 and GBM2 cell lines (which have high expression of B7H3). After transfection for $48 \mathrm{~h}$, cells were selected using $5 \mu \mathrm{g} /$ $\mathrm{mL}$ puromycin (Life Technologies, $\mathrm{CA}$ ) as suggested by the manufacturer's instructions.

\section{Cell proliferation and differentiation assay}

Cell Titer Glo Assay (Promega, Madison, WI) was used to analyze A172, GBM2, and U138 cell proliferation according to manufacturer's instructions. After differentiation, various lineage markers (GFAP, CNPase, and Tuj1) were also evaluated. A172 and GBM2 cells in serum-free medium were plated onto slides coated with poly-lysine (Sigma Aldrich, St. Louis, MO) after knockdown by B7H3 shRNA. After washing and fixation, the cells were incubated with primary mouse antibodies against human
GFAP, CNPase, and Tuj1 (1:200 each, Chemicon International Inc, Temecula, CA) for $1 \mathrm{~h}$, and then followed by secondary goat anti-mouse IgG Ab (1:200 FITC, Chemicon) and DAPI (Sigma Aldrich, St. Louis, MO) to visualize the cell nuclei for $30 \mathrm{~min}$. The cells were visualized under a fluorescence microscope (Nikon, Tokyo, Japan).

\section{Cell migration and invasion assay}

Briefly, $10^{4}$ cells were placed in Boyden chambers (BD Biosciences, NY). To rule out the effect of cell proliferation, mitomycin C (100 ng/ml, Roche, Indianapolis, IN) was added to the cells. After $2 \mathrm{~h}$, DMEM plus $10 \%$ fetal bovine serum (FBS) was placed in the lower chambers a source of chemoattractant. After $16 \mathrm{~h}$ at $37^{\circ} \mathrm{C}$, cells on the lower surface of the inserts were stained with crystal violet and counted using a light microscope. For invasion assays, inserts were coated with a thin layer of Matrigel basement membrane matrix (BD Biosciences). Cells would invade through the porous, coated membrane for $24 \mathrm{~h}$.

\section{Xenograft model in nude mice}

All animal studies were conducted in the veterinary facilities of Shanghai Institutes for Biological Sciences of the Chinese Academy of Sciences in accordance with institutional regulations. To investigate the therapeutic effect of $\mathrm{B} 7 \mathrm{H} 3$ knockdown, 6-week-old female nu/nu mice, supplied by the Experimental Animal Center, Chinese Academy of Sciences, Shanghai, were coded and then randomly divided into two groups ( $n=20,10$ for each group). A172 cells $\left(5 \times 10^{5}\right)$ in $100 \mu \mathrm{l}$ PBS were inoculated subdermally into the right thigh to generate tumors. The tumors were palpable about 10 days after injection and the diameter of the tumors was around $1 \mathrm{~cm} 20$ days after injection. The mice were treated at day 20,23 , and 26 by direct injection with B7H3 siRNA (in $100 \mu \mathrm{PBS}$ ) or with an equal volume of PBS alone (vehicle control) in a double blinded way. At day 32, the mice were sacrificed, and tumors were excised and weighed. Tumor specimens were analyzed by western blot and IHC staining.

\section{RNA sequencing, bioinformatics and statistical analysis}

A whole-genome transcriptome map could be constructed using next-generation sequencing technology. Specimens of high quality RNA (RIN $\geq 8.0)$ from A172, U138, GBM2 cell lines and corresponsive $\mathrm{B} 7 \mathrm{H} 3$ knockdown cells were used to create mRNA libraries using the Illumina TruSeq RNA Sample Preparation Kit v2. The mRNA libraries were then sequenced on the Illumina HiSeq 2500 highthroughput mode using TruSeq $^{\circledR}$ SBS v3-HS 200 cycle kit according to standard procedures generating over 
35 million 100-base pair paired-end reads (Illumina, San Diego, CA). Base calling and demultiplexing were processed using CASAVA v1.8 (Illumina, San Diego, CA), alignment was performed using TopHat 2 .

Bioinformatic analysis were performed as previously described [22, 23]. Statistical analysis was conducted with the Student $t$ test for parametric data. The results are presented as the mean \pm standard deviation (SD) of at least three independent experiments. Correlation statistics were performed using Pearson's R coefficient. Survival curves were depicted using the Kaplan-Meier plots and comparisons among groups were performed using log-rank tests in JMP9.0 (SAS Institute Inc., Cary, NC). Differences were considered significant at $P<0.05$ for all the statistical analysis (see supplementary Materials and Methods).

\section{Results}

\section{B7H3 is widely expressed in human gliomas}

Initially, gene expression of members of the $\mathrm{B} 7$ family (B7H1/CD274, B7H2/COSLG, B7H3/CD276, B7H4/ VTCNI) was assessed in the TCGA GBM dataset by analysis of mRNA levels. B7H3 was the most highly expressed among the B7 family members (Fig. S1). B7H3 was expressed at a significantly $(P<0.001)$ higher level in GBM compared with normal brain tissue (Fig. 1a). In addition, B7H3 expression was significantly higher $(P<0.05)$ in the mesenchymal subtype of GBM and gliomas with non-CpG island methylator (non-G-CIMP) phenotype $(P<0.001$, Fig. 1a).

B7H3 protein expression, assessed by western blot, was also higher in both primary and established GBM cell lines compared to adjacent non-tumor brain tissues (Fig. 1b, c). The expression of the 4Ig isoform of $\mathrm{B} 7 \mathrm{H} 3$, identified previously [16], was validated according to the splicing analysis from RNA-sequencing data (Fig. S3). B7H3 protein expression was associated with GBM tumor grade, but the level was quite heterogeneous (Fig. 1d, Fig. S2B). $\mathrm{B} 7 \mathrm{H} 3$ protein was expressed on glioma cell surfaces (Fig. 1e, S2A and S2C) and was detected by immunohistochemistry. Altogether, these results show that B7H3 is expressed in both GBM cell lines and clinical specimens, and that expression is increased in high-grade gliomas.

\section{Association of B7H3 expression with GBM tumor grade and overall survival}

Immunohistochemistry studies confirmed that B7H3 was widely expressed on gliomas and significantly associated with histological grade by the immune reactive score (IRS) (Fig. 2a, b). B7H3 mRNA expression was also positively correlated with the grade of gliomas in the GSE4290 dataset (Fig. 2c, Spearman $\rho=0.5453, P<0.001)$. The overall survival (OS) of GBM patients from Huashan Hospital of Fudan University database $(n=58)$ was significantly shorter when B7H3 was upregulated (Log rank; $P=0.042$, Fig. 2d). The median survival time was 12 vs 16 months in the high $\mathrm{B} 7 \mathrm{H} 3$ vs low $\mathrm{B} 7 \mathrm{H} 3$ group ( $n=26$ vs 32 , respectively). In addition, Rembrandt data $(P=0.005$, median cutoff, Fig. 2e) and TCGA data analyses $(P=0.026$, quantile cutoff, Fig. S4) showed a similar tendency. Hence, $\mathrm{B} 7 \mathrm{H} 3$ expression is significantly correlated with prognosis in GBM.

\section{B7H3 expression is related to the activation of genes involved in GSCs}

The functional role of $\mathrm{B} 7 \mathrm{H} 3$ remains enigmatic in malignant tumors. Bioinformatics analysis of GBM datasets showed that MYC and many other GSC-related genes were differentially expressed in $\mathrm{B} 7 \mathrm{H} 3$ high and low groups (Fig. 3a). The proximity analysis of genes involved in GSC homeostasis by Random Forest machine learning algorithm classified genes with positive and negative associations with B7H3 (CD276) expression in the TCGA dataset (Fig. 3b) (see supplementary Materials and Methods).

MYC was chosen for confirmatory studies in GBM cells, since it has been shown previously to decide tumorigenicity in GBM by our group [6]. In cell culture, higher expression of B7H3 was observed in the GBM spheres (Fig. 3c, d) in serum-free medium (SFM) with more GSC than in serum-containing medium (SCM) which induces cell differentiation $(P=0.047$, Fig. S7). Furthermore, B7H3 was co-expressed with the GSC genes A2B5 and nestin (neural stem cell markers, Fig. 3e). $\mathrm{A}^{2} \mathrm{~B}^{+}$cells (progenitor cells or GSCs [24]) and A2B5 cells were sorted to validate the differential expression of B7H3. A2B5 ${ }^{+}$cells had higher expression of B7H3 $(P=$ $0.012)$ and MYC $(P=0.025)$ than A2B5 ${ }^{-}$cells (Fig. $3 f$ and Fig. S7). Moreover, we found that the cell line of U251 and primary GBM cells, not U138 had higher expression of CD44, which was associated with B7H3 expression $(R=0.872, n=9)$ (Fig. $3 g$ and Fig. S6). According to the TCGA GBM dataset, there is a moderate correlation between CD44 and B7H3 $(R=0.435, n=$ 356) (Fig. 3h). These results indicated that upregulation of B7H3 may associate with the activation of specific GSCrelated genes.

\section{B7H3 regulates GBM cell phenotype}

The function of B7H3 in GBM was explored by knockdown using B7H3 shRNA in GBM2 and A172 cells and by overexpression by $\mathrm{B} 7 \mathrm{H} 3$ plasmid transfection into U138 

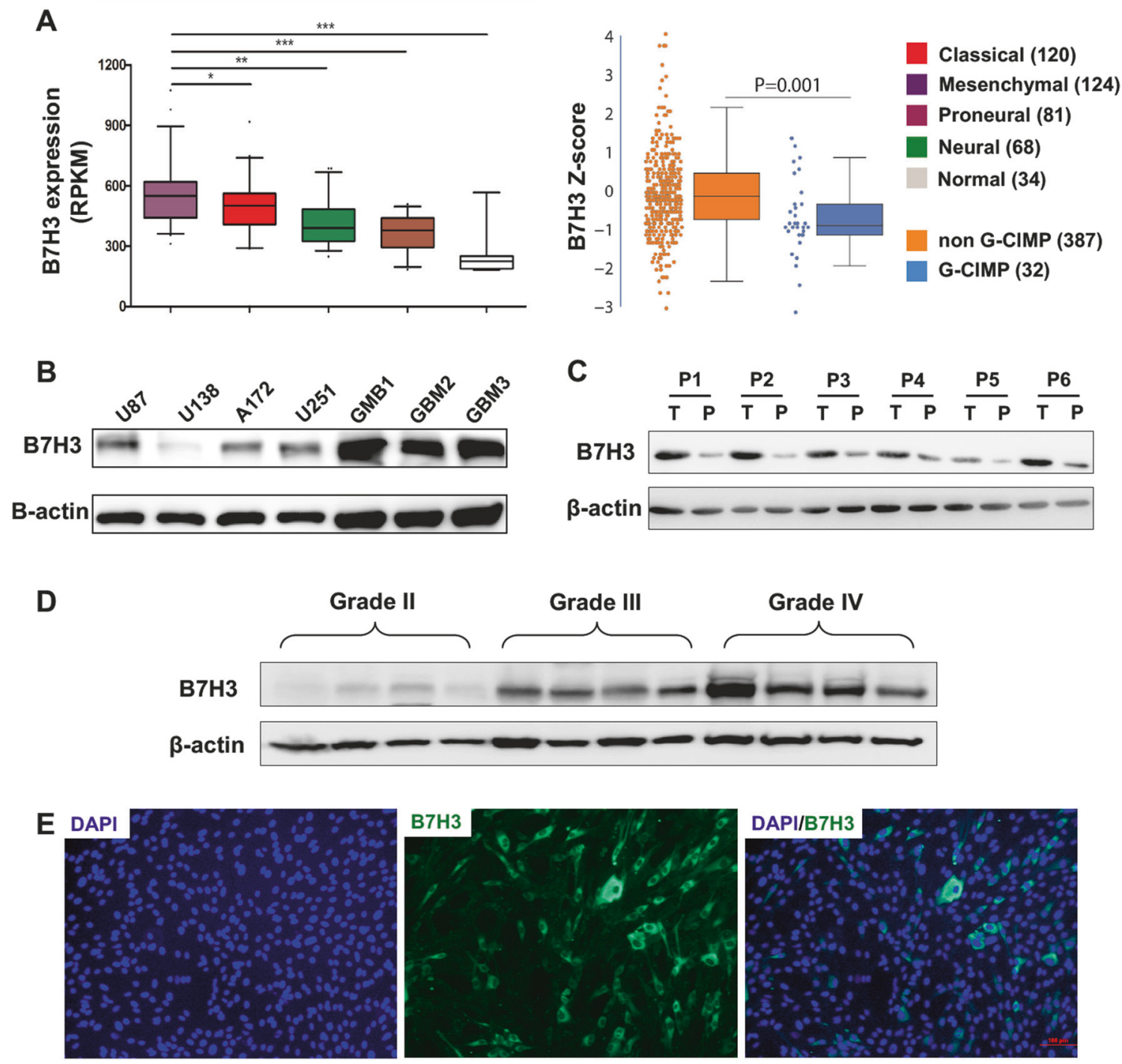

Fig. 1 Expression of B7H3 mRNA in cell lines and glioma tumor specimens. a B7H3 mRNA expression in TGCA GBM database $(n=427)$. The B7H3 gene was most highly expressed in the mesenchymal GBM subtype compared with classical, proneural or neural subtypes and normal brain tissues $(P<0.001)$. According to CIMP status, the $\mathrm{B} 7 \mathrm{H} 3$ gene was significantly more highly expressed in the non-CIMP group than the G-CIMP group $(P=0.001)$. b B7H3 protein expression in different GBM cell lines assessed by western blot. c B7H3 protein expression in glioma tumor cells (T) and adjacent non-tumor brain tissues $(\mathrm{P})$ in six different patients. $\mathbf{d}$ Representative B7H3 protein expression in different grade gliomas (grade II-IV). e Immunofluorescence staining of B7H3 (green) and DAPI nuclear staining (blue) on GBM cells (scale bar $=100 \mu \mathrm{m}$ ) cells, as verified by western blot analysis (Fig. 4a). Reduced or increased expression of $\mathrm{B} 7 \mathrm{H} 3$ did not affect the proliferation of GBM cells $(P>0.05$, Fig. S5). By contrast, the migration and invasion of $\mathrm{B} 7 \mathrm{H} 3$ knockdown GBM cell lines were decreased by $46.5 \pm 8.22 \%(P=0.001)$ and $35.4 \pm 6.39 \%(P=0.006)$, respectively, compared to control cells (Fig. 4b upper panel). Migration and invasion of the $\mathrm{B} 7 \mathrm{H} 3$ overexpressing cells increased by $34.2 \pm 8.14 \%$ $(P=0.003)$ and $25.7 \pm 7.16 \% \quad(P=0.01)$, respectively (Fig. 4b lower panel) compared to controls.

The influence of B7H3 expression on GBM cell differentiation was evaluated through monitoring cell morphology and IHC analysis of the GSC markers A2B5 and nestin, and the lineage markers GFAP, CNPase, and Tuj1 [25, 26]. After B7H3 knockdown, differentiated morphology with cell elongation and the appearance of multiple protrusions was observed. In addition, the expression of differentiation markers GFAP, CNPase, and Tuj1 were induced (Fig. 4c).

Flow cytometry analysis (Fig. 4d) demonstrated that nestin and A2B5 decreased both after serum-induced differentiation and after B7H3 knockdown. The FACS staining intensity of the GSC markers nestin $(P=0.036)$ and A2B5 were reduced in $\mathrm{B} 7 \mathrm{H} 3$ knockdown cells, although the 


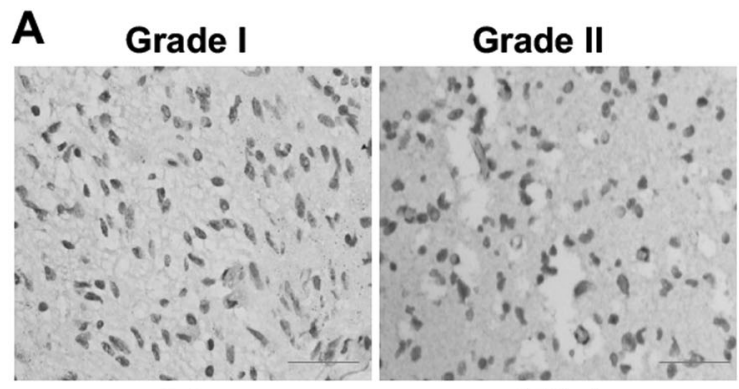

IRS=1

IRS $=6$

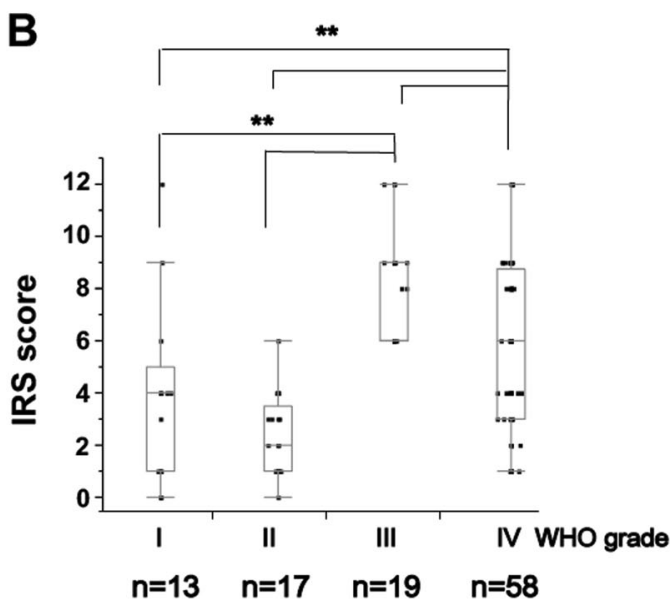

D OS of GBM patients

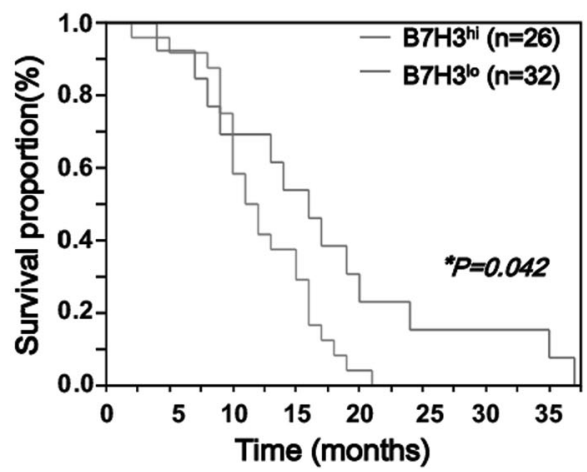

Fig. 2 Clinical significance of B7H3 in gliomas. a Representative IHC staining of B7H3 in grade I-IV gliomas (scale bar $=200 \mu \mathrm{m}$ ). b Immune reactive score (IRS) of B7H3 in different grade gliomas; Grade I: $n=13$, Grade II: $n=17$, Grade III: $n=19$, Grade IV: $n=58$. c mRNA expression level in non-tumor brain tissue and different grade gliomas from GSE4290 dataset $(n=180)$. d Kaplan-Meier survival

change of A2B5 did not reach statistical significance. Concomitantly, the level of GFAP was increased (Fig. 4d). The decrease of nestin and increase of GFAP were further confirmed with western blot analysis when $\mathrm{B} 7 \mathrm{H} 3$ was knocked down in A172 and GBM2 cells (Fig. 4e). Taken together, these studies demonstrate an association between downregulation of $\mathrm{B} 7 \mathrm{H} 3$ and the induction of differentiation in GBM cells.
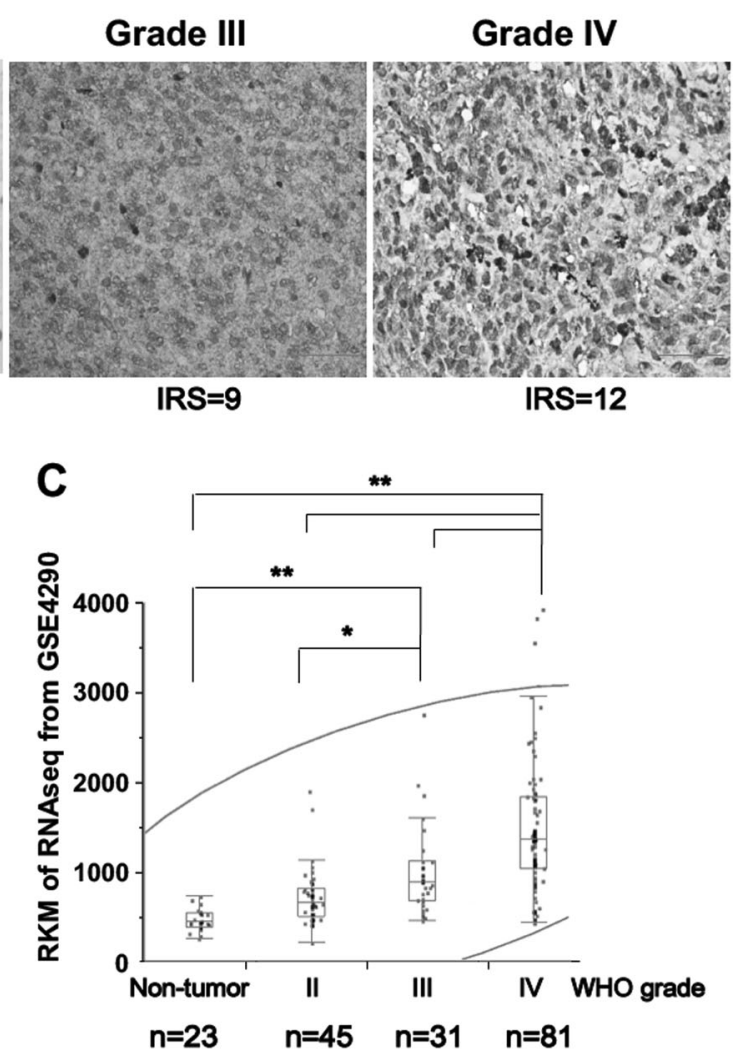

\section{E OS of GBM patients from Rembrandt}

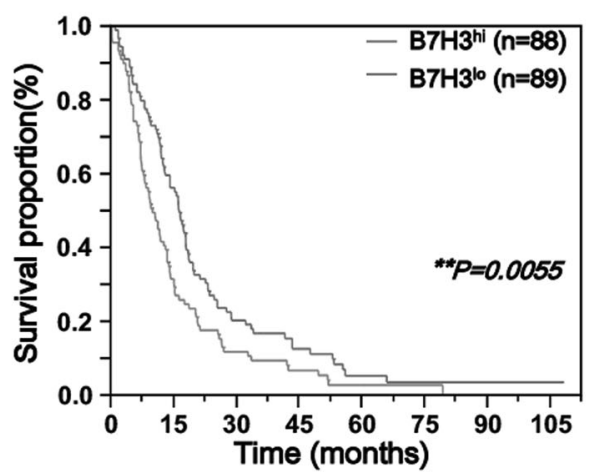

plot showing difference of overall survival (OS) between the B7H3 high (IRS $>6, n=26$ ) group and B7H3 low (IRS $\leq 6, n=32$ ) group of GBM patients $(* P=0.042)$. e: OS of the B7H3 high $(n=88)$ group of GBM patients significantly lower than B7H3 low $(n=89)$ group from Rembrandt GBM dataset $(* P=0.0055)$

\section{B7H3 knockdown activates the TGF- $\beta$ pathway and downregulates MYC expression}

To elucidate the mechanism of tumorigenicity and differentiation driven by $\mathrm{B} 7 \mathrm{H} 3$, transcriptome-wide changes after B7H3 knockdown were investigated by RNA sequencing. There were 29 genes with differential expression; nine downregulated and 20 upregulated according to $>1.5$ times 
A

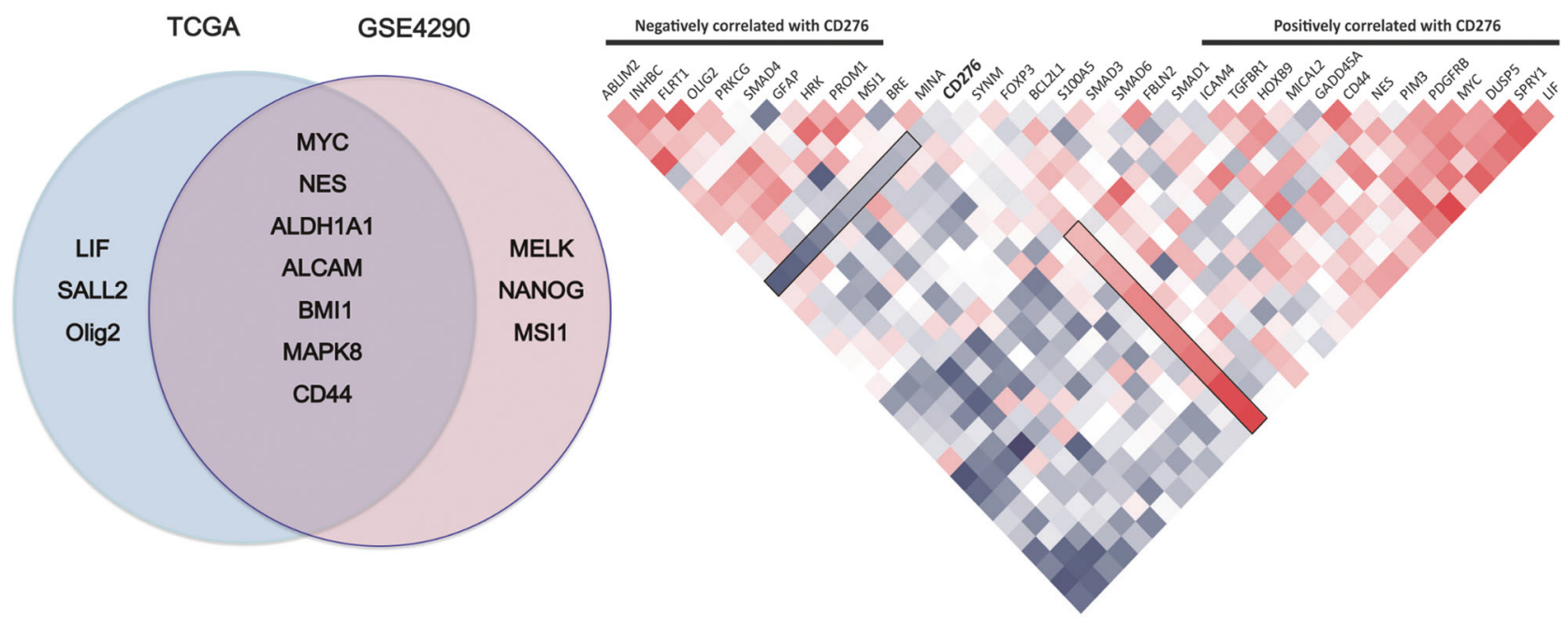

D
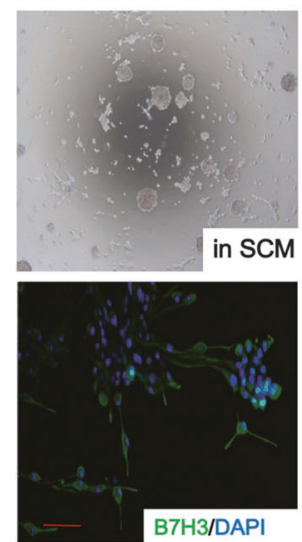

$\mathbf{F}$

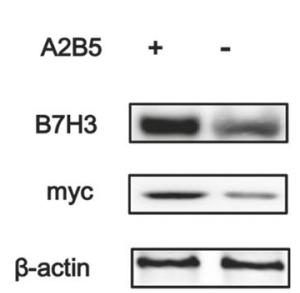

E
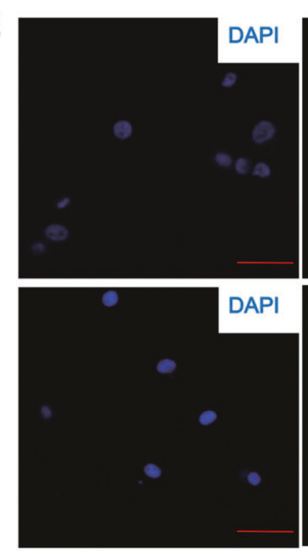

G
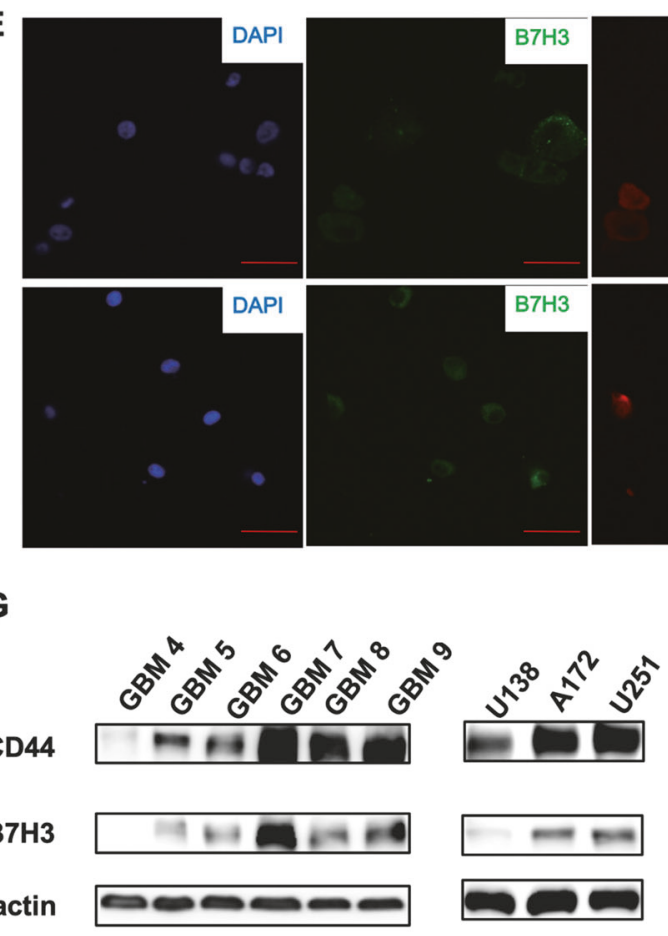
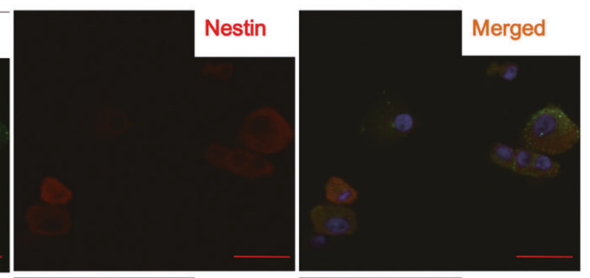

A2B5

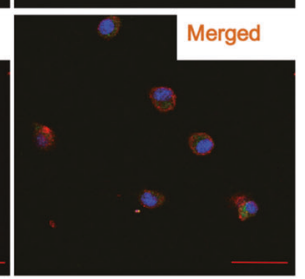

H

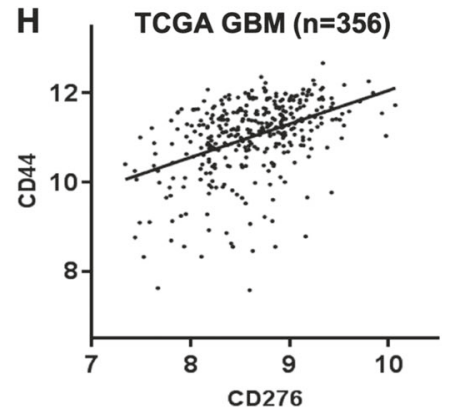

Fig. 3 Assessment of the relation between B7H3 and GSCs. a There were seven GSC-related genes which differentially expressed in $\mathrm{B} 7 \mathrm{H} 3$ high vs low groups between TCGA database and GSE4290 database. b Matrix plot analysis between $\mathrm{B} 7 \mathrm{H} 3$ and other genes according to the TCGA GBM data analysis (red means positive correlation, blue means negative correlation). c, d B7H3 was highly expressed in spheres of GBM cells in the serum-free medium (SFM) by immunofluorescence staining. e Co-expression of B7H3 (green) and nestin (red), and of

fold change of expression and consistent in all three GBM cell lines assessed (Fig. 5a). SMAD6 was the only significantly upregulated genes, when the fold change raised to 2.0. Pathway analysis of the 29 differentially expressed
B7H3 (green) and A2B5 (red). Scale bar $=50 \mu \mathrm{m})$. f The protein level of MYC and B7H3 was significantly higher in sorted $\mathrm{A} 2 \mathrm{~B}^{+}$cell than in $\mathrm{A} 2 \mathrm{~B} 5{ }^{-}$cells evaluated by western blot $(* P=0.012, P=0.025)$. g The expression of CD44, including the cell line of U251, U138, A172 and primary GBM cells was associated with B7H3 expression (Pearson $R=0.872$ ). h A moderate correlation between CD44 and B7H3 $(R=0.435, n=356)$ according to the TCGA GBM dataset

genes using the DAVID functional annotation tool showed that the P53 signaling pathway $(P=0.009)$ and the TGF- $\beta$ signaling pathway $(P=0.014)$ were the most significantly enriched (Fig. 5b). 

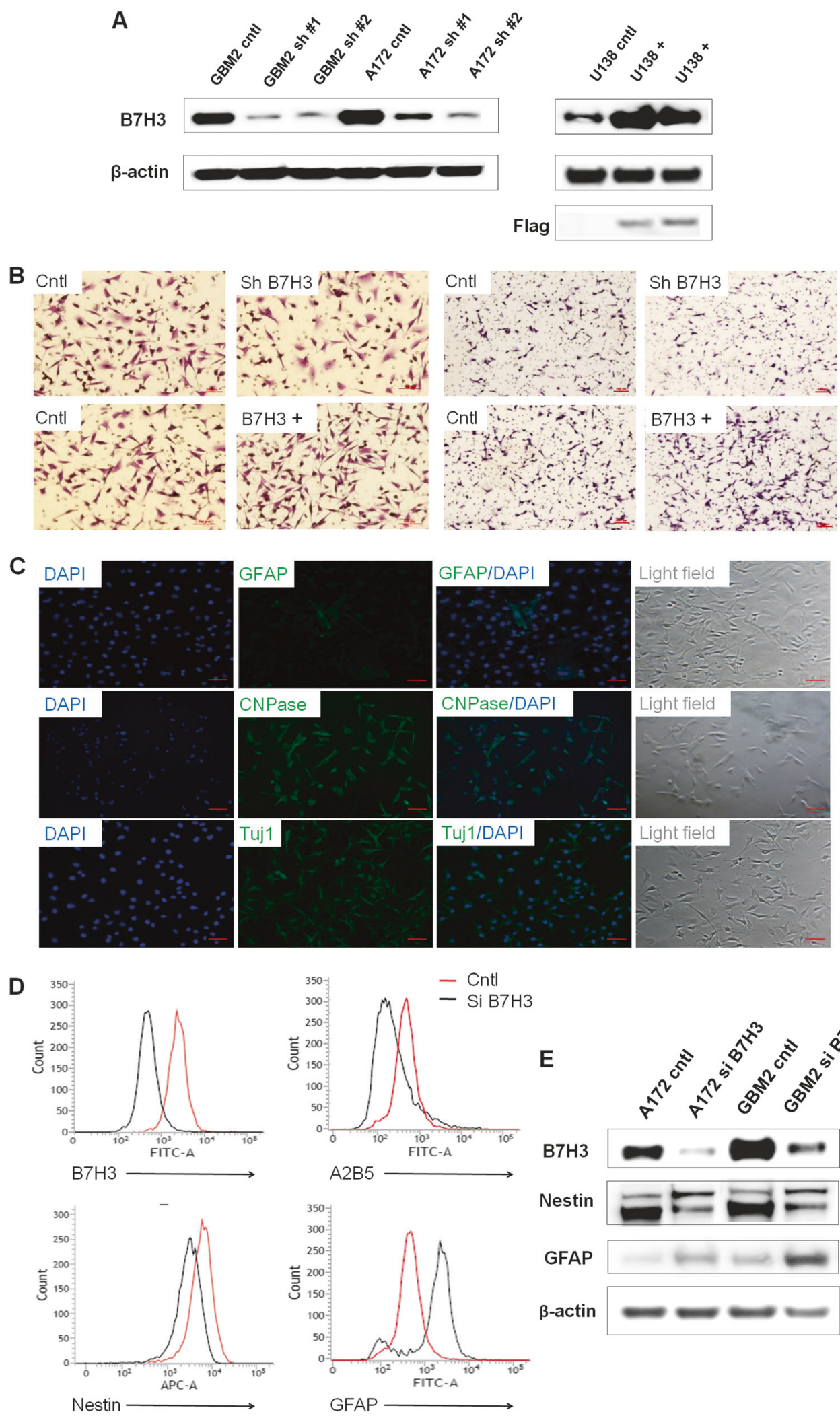

E
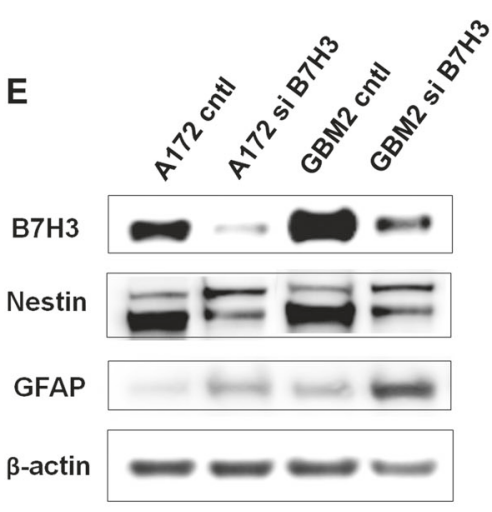

It is known that the TGF- $\beta$ pathway is involved not only in cell differentiation in GBM, but is also important to GSC [27]. To examine if differentiation induced by knockdown of B7H3 effects the TGF- $\beta$ pathway, the expression of SMAD proteins was assessed in $\mathrm{B} 7 \mathrm{H} 3$ knockdown cell lines (A172 and GBM2). A decrease of SMAD6, an 
Fig. 4 Differentiation induced by knockdown of B7H3. a Western blot confirmed the successful knockdown and overexpression of B7H3 in GBM cell lines. b The migration $(P=0.001)$ and invasion $(P=0.006)$ were decreased significantly after B7H3 knockdown (upper panel), whereas the migration and invasion increased in the $\mathrm{B} 7 \mathrm{H} 3$ overexpressed cells $(P=0.003, P=0.01$, lower panel). c Differentiation could be induced by B7H3 knockdown in GBM cells. CNPase and Tuj1 were induced significantly when B7H3 was knocked down. GFAP positive cells are scattered among the knockdown cells. DAPI: blue; CNPase: green; GFAP: green; Tuj1: green; light field: gray. Scale bar $=100 \mu \mathrm{m}$. d B7H3 was significantly reduced after knockdown (black line), and the expression of A2B5 and nestin were also more significantly reduced in the B7H3 knockdown cells than cells (red line), while the expression of GFAP was significantly increased $(P=0.018)$. e Western blot showed that B7H3 was successfully knocked down. Isoform change of nestin and the increase GFAP are observed in the B7H3 knockdown cells. There is a significant difference in mean density of nestin and GFAP between control and siRNA knockdown cells $(P<0.05)$

inhibitor of the TGF- $\beta$ pathway, was observed. The levels of the SMAD1, SMAD2, and SMAD3 seems no change (data not shown), while the levels of the phospho-SMAD1 (p-SMAD1) and SMAD4 (Fig. 5c) were significantly increased after B7H3 knockdown. Conversely, the levels of p-SMAD1 and SMAD4 were decreased when B7H3 was overexpressed in U138 cells (Fig. 5c, right lanes).

The expression of MYC, which is important in differentiation, is regulated by the TGF- $\beta$ pathway. Expression of MYC was significantly decreased after knockdown of B7H3 in A172 cells and GBM2, and was induced by B7H3 overexpression in U138 cells $(P=0.041$, Fig. 5c). These results suggested that TGF- $\beta$ pathway activation and the loss of MYC expression are downstream effects in $\mathrm{B} 7 \mathrm{H} 3$ knockdown cells, and the switch of MYC expression induced by B7H3 was dependent on TGF- $\beta$ pathway.

The bioinformatics analysis of the GBM-TCGA data ( $n=356$ ) supports the correlation between reduced $\mathrm{B} 7 \mathrm{H} 3$ expression and inhibition of the SMAD6-MYC pathway. The analysis revealed a moderate correlation between $\mathrm{B} 7 \mathrm{H} 3$ and SMAD6 (Pearson, $R=0.336$ ), B7H3 and MYC (Pearson, $R=0.387$ ), SMAD6 and MYC (Pearson, $R=0.343$; Fig. 5d). The OS time was significantly shorter among the SMAD6 upregulated group $(n=10)$ than the intermediate group $(n=149)$ (Log rank $P=0.012$, Fig. 5e) according to the Rembrandt dataset, which is consistent with a potential downstream regulatory role for $\mathrm{B} 7 \mathrm{H} 3$. Therefore, results in both cell lines and clinical analyses strongly indicated that B7H3 modulates the TGF- $\beta-M Y C$ pathway in GBM.

\section{Therapeutic targeting B7H3 in vivo}

The inhibitory effect of B7H3 knockdown on GBM was further investigated in vivo using a mouse xenograft GBM model in which A172 cells were injected directly with B7H3 siRNA on days 20, 23, and 26 (Fig. 6a). There was a significant decrease of tumor size and weight in the siRNA-treated group compared with the control group $(P=0.039$, Fig. 6b). Western blot analysis of tumor extracts demonstrated that $\mathrm{B} 7 \mathrm{H} 3$ and MYC proteins were both significantly reduced in tumors treated by B7H3 siRNA ( $P=0.044$ and $P=0.007$, Fig. 6c). IHC analysis also demonstrated the downregulation of $\mathrm{B} 7 \mathrm{H} 3$, Ki67, nestin, and MYC after B7H3 siRNA treatment (Fig. 6d). Overall, a significant reduction of GSC was induced after B7H3 siRNA treatment in vivo, and the mechanism was consistent with MYC modulation. This further supports B7H3 knockdown-induced differentiation and its theranostic potential.

\section{Discussion}

Despite recent findings and new insights into genomic changes associated with glioma, the clinical treatment improvement has been incremental in GBM patients' prognosis. B7 family members have attracted recent attention, particularly following the success of anti-PD1/PDL1 and anti-CTLA4 therapy in advanced stage melanoma and other solid tumors [7, 8]. Among the B7 family proteins, the expression of B7H3 is higher than other members in GBMs but, compared with other B7 family members, little is known about the molecular interactions and functions of B7H3.

In this study, the correlation between B7H3 gene expression and malignancy has been clearly defined utilizing different glioma datasets. This is compatible with the poorer survival being found in anaplastic astrocytoma patients with higher $\mathrm{B} 7 \mathrm{H} 3$ expression [16]. Here, we have shown that a key function of $\mathrm{B} 7 \mathrm{H} 3$, beyond immune regulation, is in GBM progression. Our studies on B7H3 function in GBM support the role of $\mathrm{B} 7 \mathrm{H} 3$ in migration and invasion, and demonstrate its new function in GBM cell differentiation rather than proliferation. All in silico analysis, immunostaining results, and RNA-sequencing data are compatible with this new function of B7H3. B7H3 knockdown also triggers nestin downregulation and, given nestin's critical role in GSC and GBM progression, its B7H3induced expression calls for further in-depth investigation.

Induction of tumor self-renewing cells differentiation has been successfully utilized in the treatment of leukemia [28] and was also studied in GBM. Hierarchies of GSC and differentiated cells in GBM have been well-demonstrated in previous studies, whereby GSC contributes to tumor recurrence, progression, and treatment resistance [29, 30]. This study discovered a significant correlation between B7H3 and the expression of GSC-related genes such as 

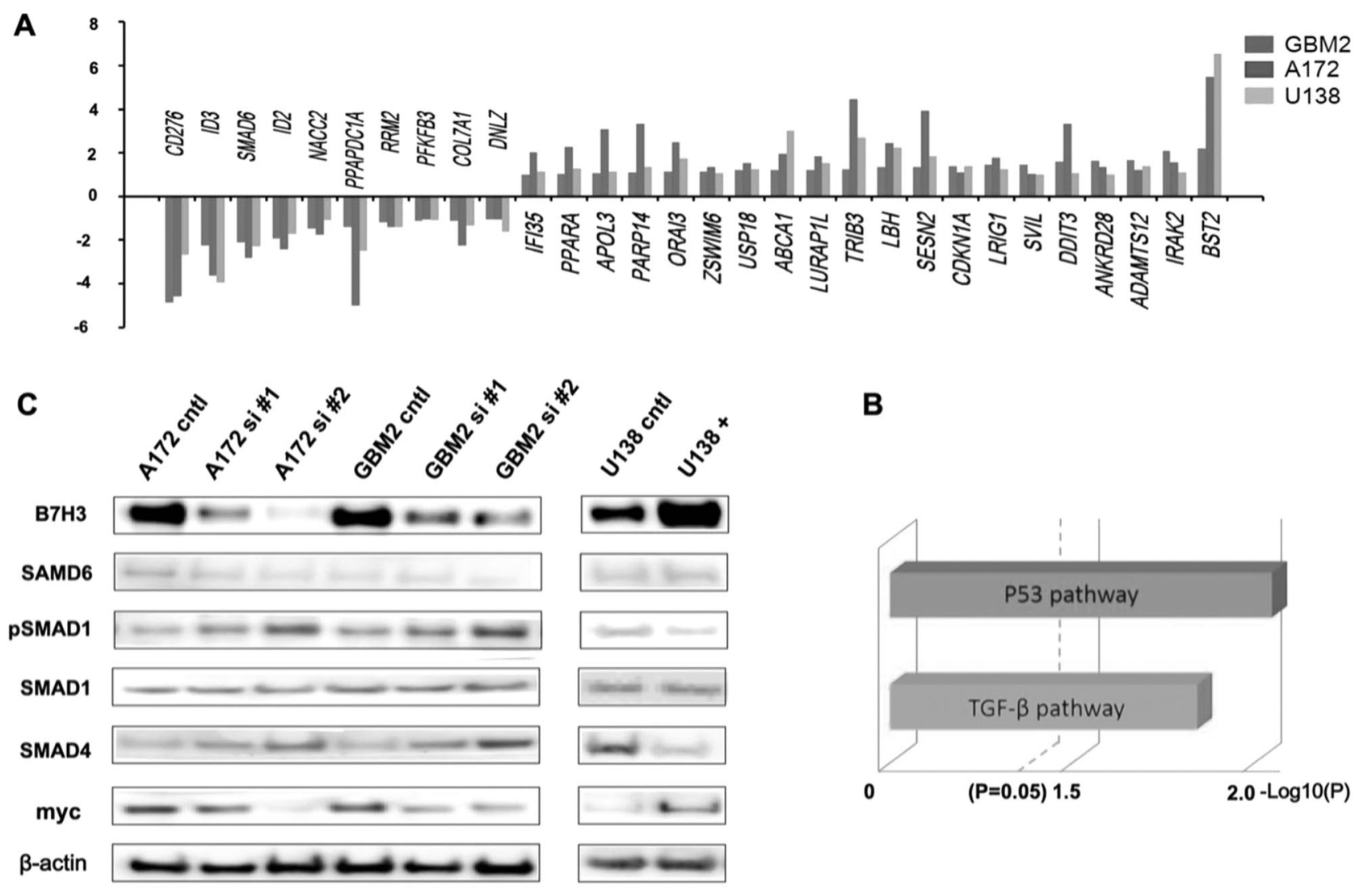

D

Pearson correlation in TCGA GBM $(n=356)$

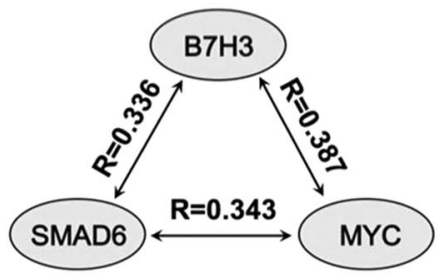

Fig. 5 Differentiation induced by B7H3 knockdown is regulated by the TGF- $\beta$ pathway and MYC. a Nine genes are downregulated while 20 genes are upregulated in $\mathrm{B} 7 \mathrm{H} 3$ knockdown cells according to the differential genes analysis of the RNAseq data (1.5-fold change). The change of these genes is consistent in GBM2 (blue), A172 (red), and U138 (green) cells. b Bioinformatic analysis of RNA-sequencing data shows that the P53 signaling pathway $(P=0.009)$ and TGF- $\beta$ pathway $(P=0.014)$ are significantly changed in the B7H3 knockdown cells. c Western blot showed that B7H3 was successfully knocked down in A172 and GBM2 cell lines by B7H3 siRNA transfection, and significantly overexpressed in U138 cells by B7H3 expression plasmid.

CD44 and A2B5, screened from the TCGA GBM dataset. $\mathrm{B} 7 \mathrm{H} 3$ is highly expressed in mouse fetal brain, suggesting B7H3's involvement in early brain development (GSE34058, data not shown). These studies point to the

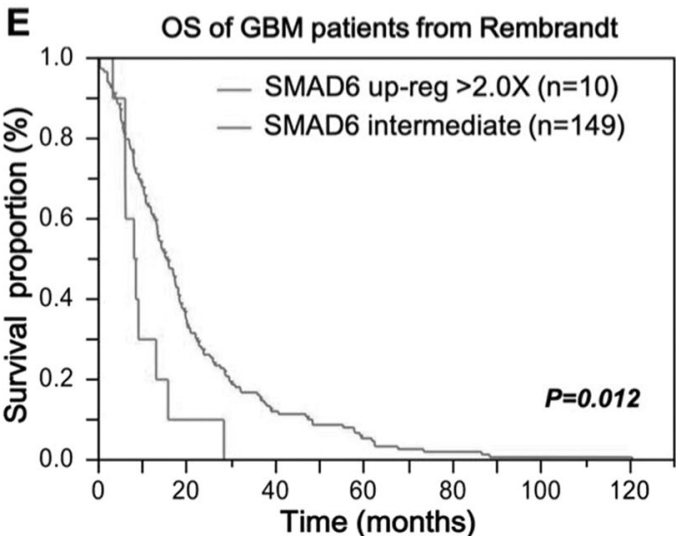

The decrease of SMAD6 and the increase of p-SMAD1 and SMAD4 were validated in the $\mathrm{B} 7 \mathrm{H} 3$ knockdown cells, corresponding to downregulation of expression of these specific genes when $\mathrm{B} 7 \mathrm{H} 3$ was overexpressed. MYC expression decreased after B7H3 knockdown, and increased after $\mathrm{B} 7 \mathrm{H} 3$ overexpression. d There was a moderate correlation between $\mathrm{B} 7 \mathrm{H} 3$ and SMAD6 (Pearson, $R=0.336$ ), B7H3 and MYC (Pearson, $R=0.387$ ), SMAD6 and MYC (Pearson, $R=0.343$ ) in TCGA GBM database $(n=356)$. e The overall survival (OS) time was significantly shorter among the SMAD6 two times upregulated group $(n=10)$ than the intermediate group ( $n=149, \log$ rank $P=0.012$ ) according to Rembrandt dataset

compelling possibility of $\mathrm{B} 7 \mathrm{H} 3$ as an oncofetal gene interwoven with stem cell functional activity.

The mechanism underlying the differentiation of brain GSCs is dependent upon many factors $[31,32]$. TGF- $\beta$ 
A

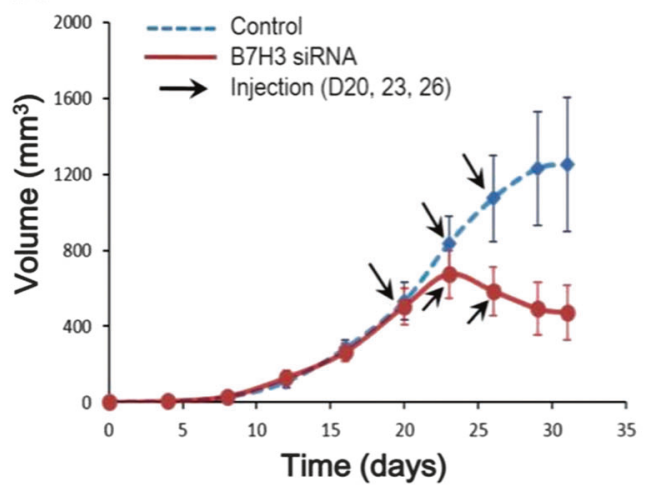

C

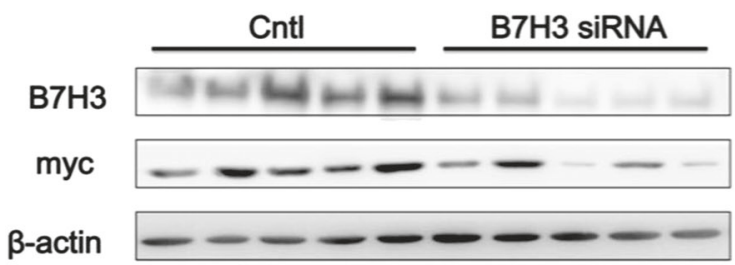

D
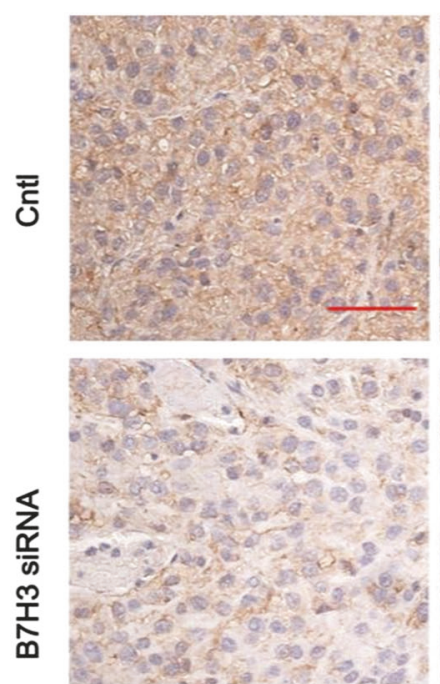

Fig. 6 Effect of direct injection of B7H3 siRNA into GBM tumors in vivo. a Tumor volume assessed of subcutaneously injected GBM cell lines (A172) followed by treatment with B7H3 siRNA. b Representative images of tumors are shown. Significant tumor shrinkage was observed after injection with B7H3 siRNA $(P=0.039)$. c MYC expression in tumors after B7H3 knockdown in vivo. Western

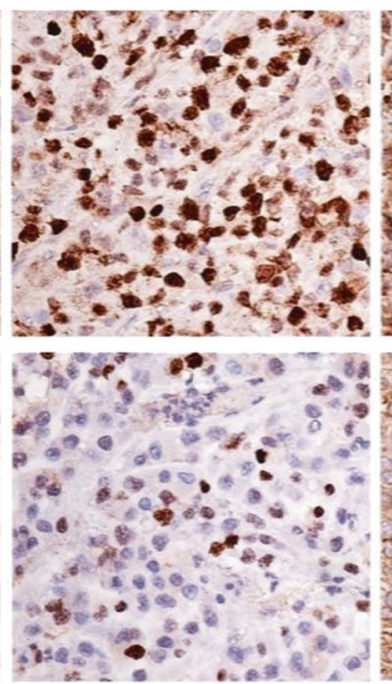

18
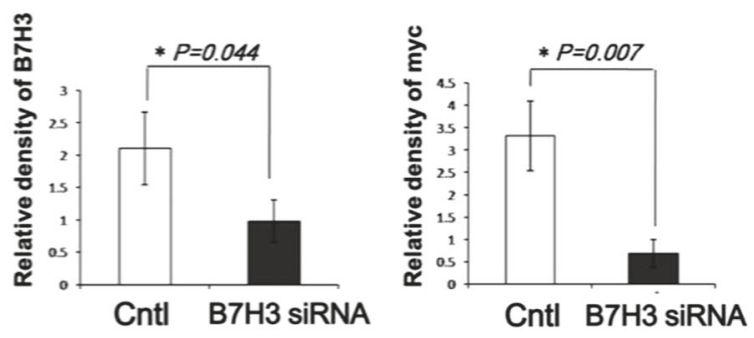

Nestin

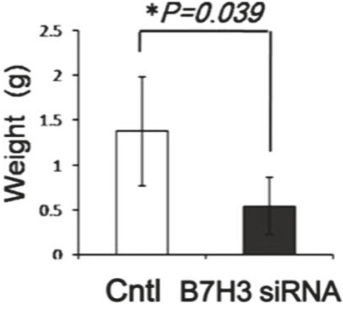

myc

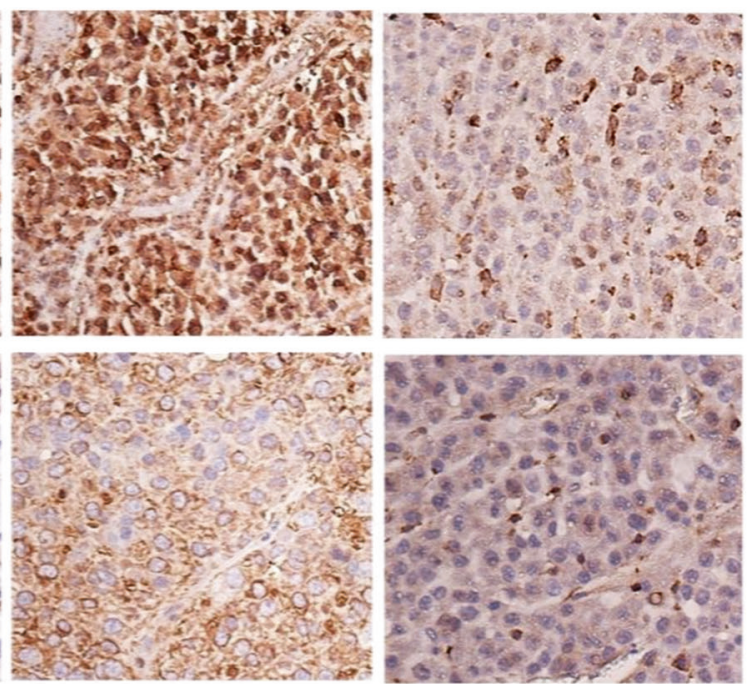

blot analysis showed that there was a significant decrease of MYC when B7H3 was knocked down $(P=0.007)$. d IHC staining of xenograft GBM tumors. Analysis showed that there was a reduction of B7H3, Ki67, Nestin, MYC, in A172 generated tumors by blocking B7H3 (lower panel, scale bar $=200 \mu \mathrm{m}$ )

differentiation effect [25]. In addition, these pathways are related to MYC, which also regulates cell differentiation $[35,36]$.

In this study, the TGF- $\beta$ pathway was identified to be closely related with $\mathrm{B} 7 \mathrm{H} 3$ expression based on RNA-sequencing data analysis, and then further validated to play a key role in this differentiation event (shown in Fig. 7). SMAD6, the negative regulator of TGF- $\beta$ pathway, 


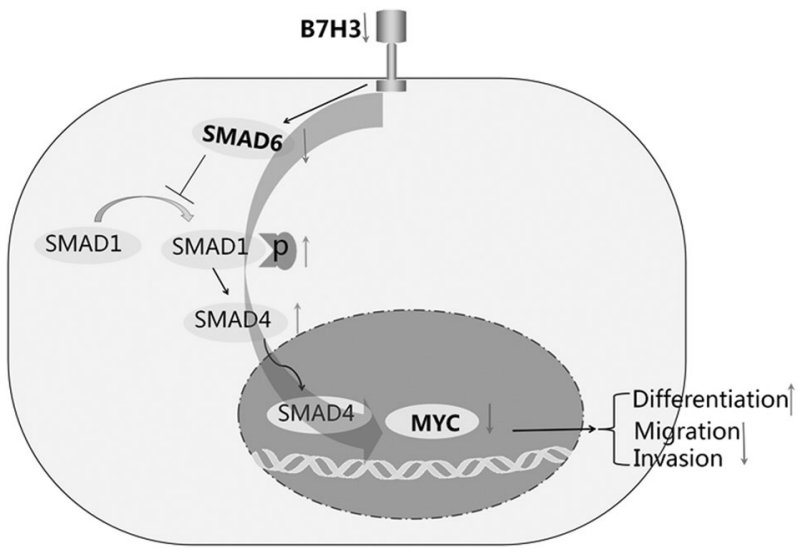

Fig. 7 Pathway model schematic. SMAD6 is an inhibitor of the TGF- $\beta$ pathway. B7H3 knockdown downregulates SMAD6, allowing activation of the TGF- $\beta$ pathway through the phosphorylation of SMAD1 and translocation of SMAD4 into the nucleus. MYC expression is decreased, thus causing differentiation and inhibition of migration and invasion

was synchronous with $\mathrm{B} 7 \mathrm{H} 3$ expression. It is proposed that the TGF- $\beta$ pathway is activated dependent on the decrease of SMAD6 and phosphorylation of SMAD1, when B7H3 is knocked down. Our in vitro and in vivo studies supported the significance of B7H3 and the TGF- $\beta$ pathway. In other reports, $\mathrm{B} 7 \mathrm{H} 3$ is shown to be involved in the activation of both the JAK2/STAT3 pathway [37] and the PI3K/AKT pathway [38].

Interestingly, MYC downregulation showed synchronous change with the decreased expression of $\mathrm{B} 7 \mathrm{H} 3$. We confirmed that activation of TGF- $\beta$ pathway can downregulate MYC, which is consistent with a previous study that TGF- $\beta$ pathway suppression can increase MYC expression [39]. In addition, our previous study demonstrated that MYC could regulate the downstream transcriptional factors [40] which are essential to GBM GSCs [6]. Our results indicate that B7H3 contributes to the undifferentiated status of GSCs through complex mechanisms.

Recently, a new monoclonal antibody to B7H3, enoblituzumab, has entered Phase I trials in selected solid tumors. Despite not knowing the receptor for the $\mathrm{B} 7 \mathrm{H} 3$ ligand or its signaling pathways, the mode of action of enoblituzumab is speculated to be (i) enhancement of adaptive T-cells and macrophage-mediated adaptive immunity (through blocking negative costimulation), (ii) direct killing of tumor cells by complement or NK cell activation, (iii) presentation of tumor antigens via $\mathrm{Fc}$-mediated interactions, or (iv) control of tumor neovascularization [41]. In our studies of in vitro and in vivo GBM cells, B7H3 demonstrates that its functional activity is tumor related and not an immune-related driven mechanism.

By contrast, we have identified the TGF- $\beta$ pathway as the downstream effector of B7H3 signaling and TGF- $\beta$ mediated regulation of glioma differentiation through MYC activation. Modulation of GBM differentiation is a potential therapeutic approach. This new mode of action of antiB7H3 therapeutics is not mutually incompatible with the proposed immunostimulatory mechanisms proposed to account for the encouraging preliminary Phase I results with enoblituzumab, but may be the major effector response [39]. All data obtained are consistent with the implication of B7H3's pivotal involvement with cell differentiation and events in carcinogenesis. Clearly, further understanding of the relationship between B7H3 and GSC differentiation and better definition of the $\mathrm{B} 7 \mathrm{H} 3$ axis is required. Regardless, B7H3 knockdown is a promising theranostic strategy to induce tumor differentiation, to be used alone or in combination with other therapeutic modalities in the future treatment of GBM.

Acknowledgements Thanks Sandy C. Hsu and Nellie Nelson for the help on sequencing and language.

Funding This work was supported by International S\&T Cooperation Program of China (2014DFA31470), China National Natural Science Foundation (81572483, 81001115), and Gonda Foundation.

\section{Compliance with ethical standards}

Conflict of interest The authors declare that they have no conflict of interest.

Ethical approval The study was approved by the independent ethics committees of Huashan Hospital, University of Fudan, Western IRB, USA, and John Wayne Cancer Institute, CA. All the informed consents were obtained from the patients.

Publisher's note: Springer Nature remains neutral with regard to jurisdictional claims in published maps and institutional affiliations.

\section{References}

1. Weller M, van den Bent M, Tonn JC, Stupp R, Preusser M, Cohen-Jonathan-Moyal E, et al. European Association for NeuroOncology (EANO) guidelines on the diagnosis and treatment of adult astrocytic and oligodendroglial gliomas. Lancet Oncol. 2017;S1470-2045:30194-8.

2. Marosi C, Preusser M. Mile stones of the last 10 years: CNS cancer. Memo. 2017;10:18-21.

3. Ceccarelli M, Barthel FP, Malta TM, Sabedot TS, Salama SR, Murray BA, et al. Molecular profiling reveals biologically discrete subsets and pathways of progression in diffuse glioma. Cell. 2016;164:550-63.

4. Seymour T, Nowak A, Kakulas F. Targeting aggressive cancer stem cells in glioblastoma. Front Oncol. 2015;5:159.

5. Auffinger B, Tobias AL, Han Y, Lee G, Guo D, Dey M, et al. Conversion of differentiated cancer cells into cancer stem-like cells in a glioblastoma model after primary chemotherapy. Cell Death Differ. 2014;21:1119-31.

6. Kozono D, Li J, Nitta M, Sampetrean O, Gonda D, Kushwaha DS, et al. Dynamic epigenetic regulation of glioblastoma tumorigenicity through LSD1 modulation of MYC expression. Proc Natl Acad Sci USA. 2015;112:E4055-64. 
7. Wolchok JD, Kluger H, Callahan MK, Postow MA, Rizvi NA, Lesokhin AM, et al. Nivolumab plus ipilimumab in advanced melanoma. N Engl J Med. 2013;369:122-33.

8. Sznol M, Chen L. Antagonist antibodies to PD-1 and B7-H1 (PDL1) in the treatment of advanced human cancer. Clin Cancer Res. 2013;19:1021-34.

9. Chapoval AI, Ni J, Lau JS, Wilcox RA, Flies DB, Liu D, et al. B7$\mathrm{H} 3$ : a costimulatory molecule for $\mathrm{T}$ cell activation and IFNgamma production. Nat Immunol. 2001;2:269-74.

10. Prasad DV, Nguyen T, Li Z, Yang Y, Duong J, Wang Y, et al. Murine B7-H3 is a negative regulator of T cells. J Immunol. 2004;173:2500-6.

11. Wang J, Chong KK, Nakamura Y, Nguyen L, Huang SK, Kuo C, et al. B7-H3 associated with tumor progression and epigenetic regulatory activity in cutaneous melanoma. J Invest Dermatol. 2013;133:2050-8.

12. Arigami T, Narita N, Mizuno R, Nguyen L, Ye X, Chung A, et al. B7-h3 ligand expression by primary breast cancer and associated with regional nodal metastasis. Ann Surg. 2010;252:1044-51.

13. Fauci JM, Straughn JM Jr, Ferrone S, Buchsbaum DJ. A review of B7-H3 and B7-H4 immune molecules and their role in ovarian cancer. Gynecol Oncol. 2012;127:420-5.

14. Loos M, Hedderich DM, Ottenhausen M, Giese NA, Laschinger M, Esposito I, et al. Expression of the costimulatory molecule B7$\mathrm{H} 3$ is associated with prolonged survival in human pancreatic cancer. BMC Cancer. 2009;9:463.

15. Zhou Z, Luther N, Ibrahim GM, Hawkins C, Vibhakar R, Handler $\mathrm{MH}$, et al. B7-H3, a potential therapeutic target, is expressed in diffuse intrinsic pontine glioma. J Neurooncol. 2013;111:257-64.

16. Lemke D, Pfenning PN, Sahm F, Klein AC, Kempf T, Warnken $\mathrm{U}$, et al. Costimulatory protein $4 \mathrm{IgB} 7 \mathrm{H} 3$ drives the malignant phenotype of glioblastoma by mediating immune escape and invasiveness. Clin Cancer Res. 2012;18:105-17.

17. Janakiram M, Shah UA, Liu W, Zhao A, Schoenberg MP, Zang $\mathrm{X}$. The third group of the B7-CD28 immune checkpoint family: HHLA2, TMIGD2, B7x, and B7-H3. Immunol Rev. 2017; 276:26-39.

18. Suh WK, Gajewska BU, Okada H, Gronski MA, Bertram EM, Dawicki $\mathrm{W}$, et al. The B7 family member B7-H3 preferentially down-regulates $\mathrm{T}$ helper type 1-mediated immune responses. Nat Immunol. 2003;4:899-906.

19. Seaman S, Zhu Z, Saha S, Zhang XM, Yang MY, Hilton MB, et al. Eradication of tumors through simultaneous ablation of CD276/B7-H3-positive tumor cells and tumor vasculature. Cancer Cell. 2017;31:501-15.

20. Hua W, Yao Y, Chu Y, Zhong P, Sheng X, Xiao B, et al. The $\mathrm{CD} 133+$ tumor stem-like cell-associated antigen may elicit highly intense immune responses against human malignant glioma. J Neurooncol. 2011;105:149-57.

21. McShane LM, Altman DG, Sauerbrei W, Taube SE. Reporting recommendations for tumor marker prognostic studies (REMARK). J Natl Cancer Inst. 2005;97:1180-4.

22. Bustos MA, Salomon MP, Nelson N, Hsu SC, DiNome ML, Hoon DS, et al. Genome-wide chromatin accessibility, DNA methylation and gene expression analysis of histone deacetylase inhibition in triple-negative breast cancer. Genom Data. 2017;12:14-6.

23. Iida Y, Ciechanover A, Marzese DM, Hata K, Bustos M, Ono S, et al. Epigenetic regulation of KPC1 ubiquitin ligase effects the NF-KB pathway in melanoma. Clin Cancer Res. 2017; 23:4831-42.

24. Ogden AT, Waziri AE, Lochhead RA, Fusco D, Lopez K, Ellis JA, et al. Identification of A2B5 + CD133-tumor-initiating cells in adult human gliomas. Neurosurgery. 2008;62:505-14.
25. Penuelas S, Anido J, Prieto-Sanchez RM, Folch G, Barba I, Cuartas I, et al. TGF-beta increases gliomainitiating cell selfrenewal through the induction of LIF in human glioblastoma. Cancer Cell. 2009;15:315-27.

26. Glasgow SM, Zhu W, Stolt CC, Huang TW, Chen F, LoTurco JJ, et al. Mutual antagonism between Sox10 and NFIA regulates diversification of glial lineages and glioma subtypes. Nat Neurosci. 2014;17:1322-9.

27. Ikushima H, Todo T, Ino Y, Takahashi M, Miyazawa K, Miyazono K. Autocrine TGF-beta signaling maintains tumorigenicity of glioma-initiating cells through Sry-related HMG-box factors. Cell Stem Cell. 2009;5:504-14.

28. Grimwade D, Mistry AR, Solomon E, Guidez F. Acute promyelocytic leukemia: a paradigm for differentiation therapy. Cancer Treat Res. 2010;145:219-35.

29. Yuan X, Curtin J, Xiong Y, Liu G, Waschsmann-Hogiu S, Farkas $\mathrm{DL}$, et al. Isolation of cancer stem cells from adult glioblastoma multiforme. Oncogene. 2004;23:9392-9400.

30. Bao S, Wu Q, McLendon RE, Hao Y, Shi Q, Hjelmeland AB, et al. Glioma stem cells promote radioresistance by preferential activation of the DNA damage response. Nature. 2006;444:756-60.

31. Massirer KB, Carromeu C, Griesi-Oliveira K, Muotri AR. Maintenance and differentiation of neural stem cells. Wiley Interdiscip Rev Syst Biol Med. 2011;3:107-14.

32. Rampazzo E, Persano L, Pistollato F, Moro E, Frasson C, Porazzi $\mathrm{P}$, et al. Wnt activation promotes neuronal differentiation of glioblastoma. Cell Death Dis. 2013;4:e500.

33. Anido J, Saez-Borderias A, Gonzalez-Junca A, Rodón L, Folch G, Carmona MA, et al. TGF-beta receptor inhibitors target the CD44 (high)/Id1(high) glioma-initiating cell population in human glioblastoma. Cancer Cell. 2010;18:655-68.

34. Nduom EK, Hadjipanayis CG, Van Meir EG. Glioblastoma cancer stem-like cells: implications for pathogenesis and treatment. Cancer J. 2012;18:100-6.

35. Guichet PO, Bieche I, Teigell M, Serguera C, Rothhut B, Rigau $\mathrm{V}$, et al. Cell death and neuronal differentiation of glioblastoma stem-like cells induced by neurogenic transcription factors. Glia. 2013;61:225-39.

36. Magri L, Gacias M, Wu M, Swiss VA, Janssen WG, Casaccia P. c-Myc-dependent transcriptional regulation of cell cycle and nucleosomal histones during oligodendrocyte differentiation. Neuroscience. 2014;276:72-86.

37. Liu F, Zhang T, Zou S, Jiang B, Hua D. B7-H3 promotes cell migration and invasion through the Jak2/Stat3/MMP9 signaling pathway in colorectal cancer. Mol Med Rep. 2015; 12:5455-60.

38. Jiang B, Liu F, Liu Z, Zhang T, Hua D. B7-H3 increases thymidylate synthase expression via the PI3k-Akt pathway. Tumour Biol. 2016;37:9465-72.

39. Chi JQ, Teng M, Yu ZH, Xu H, Su JW, Zhao P, et al. Marek's disease virus-encoded analog of microRNA-155 activates the oncogene c-Myc by targeting LTBP1 and suppressing the TGFbeta signaling pathway. Virology. 2015;476:72-84.

40. Suva ML, Rheinbay E, Gillespie SM, Patel AP, Wakimoto H, Rabkin SD, et al. Reconstructing and reprogramming the tumorpropagating potential of glioblastoma stem-like cells. Cell. 2014;157:580-94.

41. Powderly J, Cote G, Flaherty K, Szmulewitz RZ, Ribas A, Weber $\mathrm{J}$, et al. "Interim results of an ongoing phase 1, dose escalation study of mga271 (enoblituzumab), an fc-optimized humanized anti-b7-h3 monoclonal antibody, in patients with advanced solid cancer, Abstract 2015; Society for Immunotherapy of Cancer (SITC) Annual Meeting. 